\title{
Le métier d'assistant de régulation médicale (ARM) : formation et perspectives
}

\author{
Emergency Medical Call Takers and Dispatchers: Job Requirements and Professional Training
}

\author{
Y. Penverne $\cdot$ A. Ricard-Hibon \\ (C) SFMU et Lavoisier SAS 2018
}

En France, les patients sollicitant une réponse médicale d'urgence composent un numéro unique, le 15. L'appel est initialement pris en charge par un assistant de régulation médicale (ARM), il est le premier interlocuteur de l'appelant. Grâce à la maîtrise de la relation à distance, l'ARM contribue à réaliser un accueil de qualité et à instaurer une véritable relation patient à distance. Il a pour mission de localiser le patient, de circonstancier la demande et de déterminer le niveau d'urgence afin de prioriser le transfert des appels vers les médecins régulateurs [1]. Cette activité professionnelle a évolué depuis l'an 2000. Elle intègre la dynamique d'adaptation des Samu-Centres 15 et se complexifie tant sur le plan des connaissances que sur l'utilisation de nouveaux outils et de nouvelles pratiques. Les appels ont un caractère médical, parfois médico-social. Ils sont réceptionnés le plus souvent en situation ordinaire et plus exceptionnellement lors d'un événement catastrophique ou une crise sanitaire. L'ARM assure également la gestion des moyens opérationnels. Son rôle est essentiel pour la qualification initiale de l'appel. Le constat d'hétérogénéité des fonctions ou de l'organisation du métier d'ARM au sein des Samu-Centres 15 [2] et l'empirisme des formations dispensées ont contribué à la rédaction d'un référentiel métier [3] dans une volonté d'harmonisation nationale des pratiques. Il s'agit de définir, en l'absence de diplôme d'État, un cadre de formation initiale afin d'acquérir l'ensemble des savoirs et compétences nécessaires pour assurer les missions d'ARM dès la prise de fonction. Un parcours hospitalier au contact des patients et l'utilisation de techniques pédagogiques innovantes contribueraient à renforcer les connaissances générales et mettraient en perspective la réalité opérationnelle de leur métier. Si aujour-

\footnotetext{
Y. Penverne $(\bowtie)$

Samu 44, pôle Urgences, centre hospitalo-universitaire de Nantes, BP 1005, F-44093 Nantes cedex, France

e-mail : yann.penverne@chu-nantes.fr
}

\section{A. Ricard-Hibon}

Société française de médecine d'urgence,

103, boulevard Magenta, F-75010 Paris, France d'hui les ARM n'entrent pas dans le cadre réglementaire de l'obligation d'évaluation des pratiques professionnelles, il n'en demeure pas moins une nécessité dans une logique de cohérence de la réponse apportée par le Samu-Centre 15 et dans une logique de qualité et sécurité des soins. Aujourd'hui, la formation d'adaptation à l'emploi [4] définit un socle de connaissances à acquérir, elle est destinée à des agents déjà en fonction et doit être achevée à l'issue de l'année de stage qui suit la nomination dans le corps des assistants médicoadministratifs. En pratique, très peu d'ARM la valident dans les délais. Disposer d'un socle de connaissances renforcées pour assurer une écoute permanente de qualité, la gestion des moyens opérationnels, maîtriser les outils informatiques et les moyens de communication nécessite une refonte profonde de la formation des ARM à la hauteur des enjeux sanitaires confiés aux Samu-Centres 15.

\section{Références}

1. Haute Autorité de santé (HAS) (2011) Modalités de prise en charge d'un appel de demande de soins non programmés dans le cadre de la régulation médicale. https://www.has-sante.fr/portail/upload/docs/ application/pdf/2011-10/reco2clics_regulation_medicale.pdf (Dernier accès le 25 mai 2018)

2. Mardegan P (2010) Rapport de la mission DGOS relative à la modernisation des Samu. Ministère de la Santé, de la Jeunesse et des Sports. http://www.sante.gouv.fr/le-raport-mardegan.html (Dernier accès le 25 mai 2018)

3. Société française de médecine d'urgence (SFMU) (2016) Référentiel métier des assistants de régulation médicale. http://www.sfmu. $\mathrm{org} / \mathrm{upload} /$ referentielsSFMU/referentiel_arm_2016_final.pdf (Dernier accès le 25 mai 2018)

4. Ministère des Solidarités et de la Santé (2013) Arrêté du 4 juin 2013 relatif à la formation d'adaptation à l'emploi des membres du corps des assistants médicoadministratifs de la fonction publique hospitalière (branche assistance en régulation médicale). http://www.legifrance.gouv.fr/eli/arrete/2013/6/4/AFSH1315039A/ jo (Dernier accès le 25 mai 2018) 